NASA Technical Memorandum 88976

ICOMP-87-1

\title{
The Effects of Crack Surface \\ Friction and Roughness on \\ Crack Tip Stress Fields
}

$$
\begin{aligned}
& \text { (NASA-IM-88976) TEE FEFCIS CF CFACK } \\
& \text { SUEFACE FRICTICN AND FCUGHAESS CA CEACK TIE } \\
& \text { SIEESS EIELDS (KASA) } 19 \mathrm{~F}
\end{aligned}
$$

Roberto Ballarini

Case Western Reserve University

Cleveland, Ohio

and

Michael E. Plesha

Institute for Computational Mechanics in Propulsion

Lewis Research Center

Cleveland, Ohio

February 1987

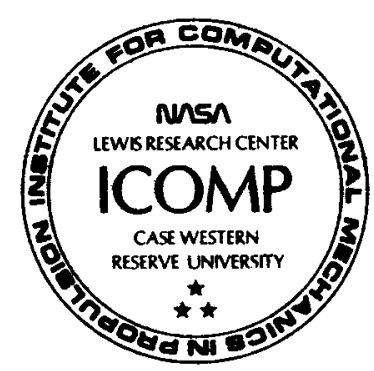




\section{ERRATA}

NASA Technical Memorandum 88976

ICOMP-87-1

The Effects of Crack Surface Friction and Roughness on Crack Tip Stress Fields

\section{Roberto Ballarini and Michael E. Plesha}

February 1987

Page 2, line 29: $n$ should be in

Page 3, equation (4): The equation should be

$$
\dot{\sigma}_{i}=E_{i j} \dot{g}_{j}^{e}
$$

Page 3, 1ine 25: The equation should be

$$
F=F\left(\sigma_{t}, \sigma_{n}, \ldots\right)
$$

Page 6, equations (17) to (20): The equations should be

$$
\begin{gathered}
c_{1} \sum_{i=1}^{N} w_{i}\left(x_{k}\right) \phi\left(t_{i}\right)=\tau_{\infty}+\sigma_{t}\left(x_{k}\right) \quad k=1, N-1 \\
c_{1} \sum_{i=1}^{N} w_{i}\left(x_{k}\right) \psi\left(t_{i}\right)=\sigma_{n}\left(x_{k}\right) \quad k=1, N-1 \\
\sum_{i=1}^{N} v_{i} \phi\left(t_{i}\right)=0 \\
\sum_{i=1}^{N} v_{i} \psi\left(t_{i}\right)=0
\end{gathered}
$$

Page 6, equation (22): The equation should be

$$
c_{1} G_{i k} \Psi_{1}=M_{k}
$$

Page 7, line 6: Change fe. to i.e. 
Page 8, equation (30): The equation should be

$$
\begin{array}{ll}
T_{1,1}=T_{1,1}+1=0.5 & \text { when } 1 \leq 1<(N-1) / 2 \\
T_{1,1}=0.75 & \text { when } 1=(N-1) / 2 \\
T_{1,1}+1=0.25 & \text { when } 1=(N-1) / 2 \\
T_{1,1}-1=0.25 & \text { when } 1=(N+1) / 2 \\
T_{1,1}=0.75 & \text { when } 1=(N+1) / 2 \\
T_{1,1}-1=T_{1,1}=0.5 & \text { when }(N+1) / 2<1 \leq N-1
\end{array}
$$

Page 9, equation (32): The equation should be

$$
\left(\tan \frac{\theta}{2}\right)=0.25 \frac{K_{I}}{k_{I I}}-0.25\left(\frac{K_{I}^{2}}{k_{I I}^{2}}+8\right)^{1 / 2}
$$


THE EFFECTS OF CRACK SURFACE FRICTION AND ROUGHNESS ON

\title{
CRACK TIP STRESS FIELDS
}

Roberto Ballarini*

Case Western Reserve University

Department of Civil Engineering

Cleveland, Ohio 44106

\author{
and \\ Michael E. Plesha** \\ Institute for Computational Mechanics in Propulsion \\ Lewis Research Center \\ Cleveland, Ohio 44135
}

\begin{abstract}
SUMMARY
A model is presented which can be used to incorporate the effects of friction and tortuosity along crack surfaces through a constitutive law applied to the interface between opposing crack surfaces. The problem of a crack with a saw-tooth surface in an infinite medium subjected to a far-field shear stress is solved and the ratios of Mode-I stress intensity to Mode-II stress intensity are calculated for various coefficients of friction and material properties. The results show that tortuosity and friction lead to an increase in fracture loads and alter the direction of crack propagation.
\end{abstract}

\section{INTRODUCTION}

The essential ingredients which enter a Linear Elastic Fracture Mechanics (LEFM) analysis are stress intensity factors and fracture toughness. These quantities can be used to predict fracture initiation loads as well as the direction of crack propagation. Stress intensity factors have been obtained for a large variety of loadings and specimen geometries. In most of these analyses cracks are modeled as discontinuities possessing smooth and frictionless surfaces. For Mode-I type loadings the assumption that the surfaces of a crack are smooth and frictionless is quite satisfactory, but for Mode-II loadings or mixed-mode loadings this assumption may lead to erroneous results if the crack surfaces are tortuous and/or offer frictional resistance to sliding. For such problems the effects of tortuosity and friction must be accounted for in order to capture the physics of the phenomena and to correctly assess the stress intensity factors. This can be seen by considering the crack shown in figure 1(a). Let us assume that this crack was modeled as being smooth and frictionless. If a far-field shear stress (an apparent Mode-II loading) is applied to this crack, the model would predict a zero Mode-I stress intensity factor. In reality, the Mode-I stress intensity factor is not zero, since the displacement of a point along the surface of the

*Work funded by Cooperative Agreement NCC 3-46.

* Work funded under Space Act Agreement C99066-G and U.S. Army Research Office Grant DAAL03-86-K-0134; presently at University of Wisconsin, Department of Engineering Mechanics, Madison, Wisconsin 53706. 
crack has both a normal and a tangential component. The tangential component will result in a Mode-II stress intensity, while the normal component will result in a Mode-I stress intensity.

Cracks of the type shown in figure 1 (a) have been observed in many polycrystalline and aggregate materials. Some examples are metal, ceramic, concrete and brittle geo- and bio-materials such as rock and bone, respectively. Methods of analys is for mixed-mode fracture due to tortuosity are primitive. clech et al. (ref. 1) have observed and analyzed tortuous cracks propagating along the interface between cancellous bone and the cement which binds an artificial joint to it. Their analysis considered failure under Mode-I loading, where the tortuosity was modeled as a no-slip interface. The question arises as to what would be the most efficient way to model the problem if the structure is subjected to mixed-mode loading. One approach would be to model the geometry of the crack surfaces explicitly in a finite-element (or boundary element) mesh, assign a coefficient of friction to the crack surfaces, and to solve the nonlinear stress analysis problem. This technique is obviously cumbersome. Concrete is another material for which friction and tortuosity play a significant role during crack propagation (refs. 2 to 5). Recently, Ingraffea et al. (ref. 5), using the finite element method, have modeled frictional interlock effects in concrete as distributions of normal and shear stresses along the interface between opposing crack surfaces. Their model, however, does not account for the previously discussed coupling between crack sliding displacements and crack opening displacements. Riggs and Powell present a rough crack model for the analys is of concrete in reference 6 . This model possesses many desirable features compared to previous attempts at rough crack modeling but is complex and has a number of empirical constants that require specification.

In this paper a model is presented which can be used to characterize the effects of friction and tortuosity along crack surfaces $n$ an efficient and realistic manner. In this model the crack surfaces are assumed to be globally smooth, and the roughness and friction are incorporated through a constitutive law at the interface between opposing crack surfaces. This constitutive law contains implicitly the coupling between the tangential and normal components of the displacement of a point on the interface (this phenomenon is referred to as dilatancy), and can therefore give rise to Mode-I stress intensity factors even when the applied loading is apparently Mode II.

\section{CONSIITUTIVE LAW}

In this section we review the development of analytic relations between increments of crack surface traction and crack sliding and opening displacements. This theory was fully developed in (ref. 7) and is analogous to the theory of continuum elasto-plasticity. The surface between two bodies coming into contact is assumed to be globally smooth as shown in figure 2(a). Attention is focused on a typical pair of initially adjacent points on the contact surface as -shown in figure 2(b); these points are shown separated for clarity and the roughness that the surfaces have is not shown and will be considered subsequently.

The traction that is supported by the crack surface at the contact point in question has tangential and normal components which are denoted by ot and $\sigma_{n}$, respectively. The crack sliding and opening displacements at the contact point pair are denoted by $g_{t}$ and $g_{n}$, respectively, where 


$$
\begin{aligned}
& g_{t}=u_{x}^{+}-u_{x}^{-} \\
& g_{n}=u_{y}^{+}-u_{y}{ }^{-}
\end{aligned}
$$

In equations (1) and (2), $u_{x}$ and $u_{y}$ are horizontal and vertical displacements (i.e., displacements tangential and normal to the global crack surface), respectively, while superscripts + and - denote points on the contact surface associated with the upper and lower bodies of material, respectively.

It is assumed that the relative displacements of the crack surface are additively composed of reversible (elastic) and irreversible (plastic) parts

$$
g_{i}=g_{i}^{e}+g_{i}^{p} \quad 1=t, n
$$

where superscripts $e$ and $p$ denote the elastic and plastic parts of the deformation. In equation (3), the plastic part represents the permanent deformation due to sliding (and perhaps damage), which has components in the normal direction (due to dilatancy) as well as in the tangential direction. The elastic part represents deformations that occur independently of sliding such as, for example, presliding deformability. Such recoverable deformations have been observed in a large variety of experiments between materials such as metals, ceramics, and rocks (refs. 8 to 10). The stress that the interface supports is assumed to be related to the elastic part of equation ( 3 ) by

$$
\sigma_{1}=E_{i j} \dot{g}_{j}^{e}
$$

where the summation convention is applied to repeated indices and $E_{i j}$ are the interface stiffnesses, the determination of which will be addressed shortly.

To prescribe a method for determining the plastic displacements, it is necessary to assume that (1) a scalar valued slip function $F=F\left(\sigma_{t}, \sigma_{n}, \ldots\right.$ can be defined such that $F<0$ corresponds to presiliding conditions, $F=0$ corresponds to sliding and $F>0$ is not possible, and (2) there exists a linear relationship between increments of traction and plastic deformation. Under these assumptions, it can be shown (ref. 11) that

$$
\mathrm{dg}_{i}^{p}= \begin{cases}0 & \text { if } F<0 \text { or } d F<0 \\ \lambda \frac{\partial G}{\partial \sigma_{i}} & \text { if } F=d F=0\end{cases}
$$

where $G$ is the slip potential whose gradient gives the direction of the plastic deformation and $\lambda$ is a nonnegative scalar that gives its magnitude. Expressions for $F$ and $G$ can be obtained by considering the idealized roughness shown in figure $2(\mathrm{c})$. If the friction on the active asperity surface is governed by Coulomb's law, then $\left|\sigma_{1}\right| \leq-\mu \sigma_{2}$ where $\mu$ is the coefficient of friction, $\sigma_{1}$ and $\sigma_{2}$ are the tangential and normal stresses on the asperity surface and the convention that compressive stresses are negative is employed. By stress transformation, this equation can be expressed in terms of the global 
stresses of and on (ref. 7). This procedure leads to the following forms for the slip function and slip potential

$$
\begin{aligned}
F=\mid \sigma_{n} \sin \alpha_{k} & +\sigma_{t} \cos \alpha_{k} \mid+\mu\left(\sigma_{n} \cos \alpha_{k}-\sigma_{t} \sin \alpha_{k}\right) \\
G & =\left|\sigma_{n} \sin \alpha_{k}+\sigma_{t} \cos \alpha_{k}\right|
\end{aligned}
$$

where $k=R$ or $L$ depending upon which asperity surface is active (fig. 2(c)).

Combining equations (3) to (5) leads to the constitutive relation

$$
d \sigma_{i}=E_{i j}^{e p} d g_{j}
$$

where

$$
E_{i j}^{e p}=E_{i j} \text { if } F<0 \text { or } d F<0
$$

or

$$
E_{i j}^{e p}=E_{i j}-\frac{\frac{\partial F}{\partial \sigma_{p}} E_{i q} E_{p j} \frac{\partial G}{\partial \sigma_{q}}}{\frac{\partial F}{\partial \sigma_{p}} E_{p q} \frac{\partial G}{\partial \sigma_{q}}-H} \text { if } \quad F=d F=0
$$

and $H$ is a hardening or softening parameter that can account for the damage of crack surface roughness. In this paper we take $H$ equal to zero.

The following remarks about the constitutive law should be noted:

(1) Equation (8) is an explicit relation between increments of crack surface tractions and the resulting crack sliding and opening displacements. Unlike some other theories for dilatant crack surface problems (ref. 12) in which equation (8) is postulated at the outset and the $E_{i j}^{e p}$ (particulariy the $E_{t n}^{e p}$ and $E_{n t}^{e p}$ ) are determined either experimentally or according to some ad hoc rule, this theory provides an explicit and unambiguous method for determining $E_{i j}^{e p}$

(2) The theory presented in this section is essentially a "continuum" theory of friction except that we refer to continuum in the sense of continuous area rather than continuous volume. The traction components ot and on entering into the theory are the average or macroscopic stresses that the crack surface supports rather than the exact stresses. Furthermore, the global crack surface is smooth and the effects of roughness (e.g., dilatancy) are built into the slip rule and slip potential.

(3) The use of the decomposition in equation (3) in conjunction with equation (4) can be considered a relaxation of compatibility of crack surface displacements consisting of impenetrability and presliding stick. In order to render the violation of compatibility insignificant, it is necessary to make the interface stiffnesses $E_{i j}$ relatively large compared to the stiffness of the adjacent medium. In this investigation, we take $E_{i j}=P \delta_{i j}$, 
where $\delta_{i j}$ is the Kronecker delta and $P$ is a penalty number. The penalty number has no physical significance and is chosen based on computational convenience. Values of $P$ that are two to four orders of magnitude greater than the shear modulus of the adjacent medium were used and provided solutions in which incompatibilities were extremely small. Larger values of $p$ sometimes gave numerical difficulties due to ill-conditioning while smaller values of $P$ gave sightiy excessive incompatibility.

(4) It is assumed in this research that the amount of tangential siiding is small enough so that the asperity peak of one surface does not override that of the other surface. For situations in which this is not the case, the theory can be supplemented with an additional set of conditions as discussed in reference 7 .

\section{COMPLIANCE MATRIX}

The problem which will be solved to demonstrate the significance of friction and tortuosity is shown in figure 1. The singular integral equations that govern this problem are

$$
\begin{gathered}
\frac{2 G}{\pi(k+1)} \int_{-1}^{1} \frac{f(t) d t}{t-x}=\tau_{\infty}+\sigma_{t} \quad-1<x<1 \\
\frac{2 G}{\pi(k+1)} \int_{-1}^{1} \frac{e(t) d t}{t-x}=\sigma_{n} \quad-1<x<1
\end{gathered}
$$

where $\tau_{\infty}$ is the far-field shear stress, $\sigma_{t}$ and $\sigma_{n}$ are the shear and normal stresses applied to the crack surfaces, $G$ is the shear modulus, $k=3-4 v$ for plane strain, $v$ being Poisson's ratio, and $f(t)$ and $e(t)$ are the dislocation densities, which are related to the crack sliding and crack opening displacements by

$$
\begin{aligned}
& u_{x}^{+}-u_{x}^{-}=\int_{x}^{1} f(t) d t=g_{t} \\
& u_{y}^{+}-u_{y}^{-}=\int_{x}^{1} e(t) d t=g_{n}
\end{aligned}
$$

Because the crack is closed at the endpoints the dislocation densities must satisfy the following conditions

$$
\int_{-1}^{1} f(t) d t=\int_{-1}^{1} e(t) d t=0
$$

Since ot and $\sigma_{n}$ depend on the relative displacements between the crack surfaces, the singular integral equations are nonlinear. In order to solve these equations efficiently, a compliance matrix will be developed that relates the stresses at the collocation points arising from the applied loads to the values of the opening (or sliding) of the crack at the integration points. The method relies on the numerical procedure developed by Gerasoulis (ref. 13), 
which is used to reduce the integral equations to a system of algebraic equations for unknown values of $e(t)$ and $f(t)$ at discrete points in the interval $[-1,1]$ : With the aid of the compllance matrix, it will be possible to obtain the crack opening and crack sliding displacements for a given stress distribution on the crack surfaces by solving simultaneous algebraic equations and hence, the need to repeatedly solve the integral equations and integrate the dislocation densities is eliminated. The procedure for obtaining the compliance matrix is described in detall in reference 14 and is only reviewed here. Following (ref. 13), $f(t)$ and $e(t)$ are expressed by

$$
f(t)=\phi(t)\left(1-t^{2}\right)^{-1 / 2} \quad e(t)=\psi(t)\left(1-t^{2}\right)^{-1 / 2}
$$

and $\varphi(t)$ and $\psi(t)$ are approximated as piecewise quadratic on the interval $[-1,1]$. The result is that the integral equations are reduced to a system of algebraic equations through the quadrature formulas given in (ref. 13). The results can be written symbolically as

$$
\begin{gathered}
c_{1} \sum_{i=1}^{N} w_{i}\left(x_{k}\right) \phi\left(t_{i}\right)=\tau_{\infty}+\sigma_{t}\left(x_{k}\right) \quad k=1, N-1 \\
c_{1} \sum_{i=1}^{N} w_{i}\left(x_{k}\right) \psi\left(t_{i}\right)=\sigma_{n}\left(x_{k}\right) \quad k=1, N-1 \\
\sum_{i-1}^{N} v_{i} \phi\left(t_{i}\right)=0 \\
\sum_{i-1}^{N} v_{i} \psi\left(t_{i}\right)=0
\end{gathered}
$$

where $c_{1}=2 G / \pi(k+1), N$ is the number of integration points, $N-1$ is the number of collocation points, and the weights $w_{j}\left(x_{k}\right)$ and $v_{j}$, as well as the collocation points $x_{k}$ and integration points $t_{j}$ are given in (ref. 13) (the locations of the integration and collocation points are shown schematically in figure $3(a))$. These equations can be written as

$$
\begin{aligned}
& c_{1} G_{i k} \phi_{i}=L_{k} \\
& c_{1} G_{i k} \psi_{j}+M_{k}
\end{aligned}
$$

where matrices [L] and [M] represent the nodal values of the stresses along the crack surfaces and the crack closure conditions. To obtain the compliance matrices for the crack the inverse of matrix [6] is obtained, and the product of this matrix and $\left(1-t^{2}\right)^{-1 / 2}$ is integrated term by term to obtain a matrix $[\widetilde{c}]$ which will be called the compliance matrix for this particular geometry. This matrix will relate the values of crack sliding displacements (or crack opening displacements) at the integration points to the values of the shear (or normal) stresses at the collocation points, that is 


$$
\begin{gathered}
\tilde{c}_{i k}\left(\tau_{x}+\sigma_{t}\left(x_{k}\right)\right)=\left(u_{x}^{+}-u_{x}^{-}\right) t_{j}=\left(g_{t}\right)_{t_{i}} \\
\tilde{c}_{i k} \sigma_{n}\left(x_{k}\right)=\left(u_{y}^{+}-u_{y}^{-}\right)_{t_{i}}=\left(g_{n}\right) t_{i}
\end{gathered}
$$

where $\tilde{\mathrm{C}}_{i k}$ is a rectangular matrix with dimensions $(N, N-1)$. We wish to point out that because of the technique employed to determine the compliance matrix, the locations at which the crack opening and crack sliding displacements are determined (ie., the integration points) are different from the locations of crack surface tractions (i.e., the collocation points). Furthermore, the number of displacement points is one greater than the number of stress points. In order to eliminate the computational difficulties associated with differing numbers of collocation and integration points, we present in the following section a method for rendering the compliance matrix square.

The stress intensity factors are proportional to the values of the functions $\phi$ and $\psi$ at the endpoints (ref. 13) and can be obtained by premultiplying matrices [L] and [M] by the inverse of matrix [G]. The details of the calculations can be found in references 13 and 14 . We note that the compliance matrix for shear loading is the same as that for normal loading. This is because the Green's functions for the two problems are the same.

\section{NUMERICAL SOLUTION}

Before writing equations (23) and (24) in matrix form, it is necessary to make the compliance matrix square. Because the number of integration points is odd (ref. 13), there is an integration point at the center of the crack. By eliminating the equation corresponding to this point from equation (23), the number of displacement points is reduced by one and the compliance matrix becomes square. Following this procedure the location of the displacement and stress points become as shown in figure $3(\mathrm{~b})$. The compliance matrix is now denoted by $[\mathrm{C}]$ and is given by

$$
[C] \equiv C_{i j}=\left\{\begin{array}{lll}
\tilde{c}_{i j} & \text { if } & 1<(N+1) / 2 \\
\tilde{c}_{(1+1) j} & \text { if } & 1 \geq(N+1) / 2
\end{array}\right.
$$

where $i, j=1,2, \ldots, N-1$. The modified forms of equations (23) and (24) can now be written collectively in incremental form as

where

$$
\left\{\begin{array}{l}
d g_{t} \\
d g_{n}
\end{array}\right\}=\left\{\begin{array}{c:c}
c & 0 \\
\hdashline 0 & c
\end{array}\right)\left\{\begin{array}{c}
d \tau_{\infty}+d \sigma_{t} \\
\hdashline d \sigma_{n}
\end{array}\right\}
$$

$$
\begin{aligned}
& \left\{d g_{t}\right\}=\left[\left(d g_{t}\right)_{t_{1}}, \ldots,\left(d g_{t}\right)_{t_{N-1}}\right]^{\top} \\
& \left\{d \sigma_{t}\right\}=\left[\left(d \sigma_{t}\right)_{x_{1}}, \ldots,\left(d \sigma_{t}\right)_{x_{N-1}}\right]^{\top}
\end{aligned}
$$


with similar definitions for $\left\{d g_{n}\right\}$ and $\left\{d \sigma_{n}\right\} . d \tau_{\infty}$ is a vector with $N-1$ components, each equal to the increment in the applied loading.

Although the number of displacement points and stress points are now the same, the locations of these points are different as shown in figure $3(b)$. The constitutive law given by Eq. (8), however, relates the crack surface stresses at a point to the displacements at the same point. The crack displacements at the stress points can be expressed in terms of those at the integration points by interpolation. For example, the relation between the crack sliding displacements at the stress points and the collocation points is given by

$$
\left\{\mathrm{dg}_{\mathrm{t}}\right\}_{x_{i}}=[\mathrm{T}]\left\{\mathrm{dg}_{\mathrm{t}}\right\}_{\mathrm{t}_{i}}
$$

where the coefficients of [T] are zero except for

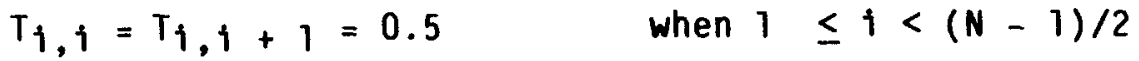

$$
\begin{aligned}
& T_{i, i}=0.75 \quad \text { when } i=(N+1) / 2
\end{aligned}
$$

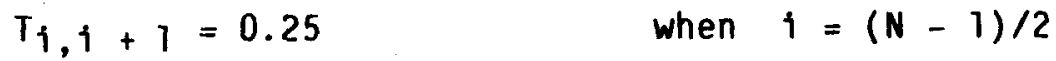

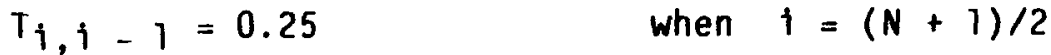

$$
\begin{aligned}
& T_{i, i}=0.75 \quad \text { when } i=(N+1) / 2
\end{aligned}
$$

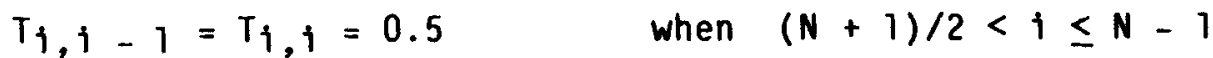

Combining equations (8), (26), and (29) leads to

$$
\left[[1]+\left\{\begin{array}{ll}
c & 0 \\
0 & c
\end{array}\right\}\left[E^{e P}\right]\left\{\begin{array}{ll}
T & 0 \\
0 & T
\end{array}\right\}\right]\left\{\begin{array}{l}
d_{t} \\
d g_{n}
\end{array}\right\}=\left\{\begin{array}{ll}
c & 0 \\
0 & c
\end{array}\right\}\left\{\begin{array}{l}
d \tau \\
0
\end{array}\right\}
$$

which is a system of simultaneous nonlinear algebraic equations to be solved for the incremental crack opening and silding displacements. By taking small increments in far-field shear loading, good convergence was attained in the numerical work, where $\left\{\mathrm{EP}^{\mathrm{eP}}\right.$ was computed at each step using the displacementstress configuration from the previous step and treating equation (31) as linear within each load increment.

\section{RESULTS}

The problem shown in figure 1 was solved for the various combinations of material properties listed in table I. Figures 4 to 6 are plots of stress intensity factors as functions of the far-field shear stress for several values of the uniform asperity angle $\alpha$. It can be seen from these figures that as $\alpha$ increases, $K_{I}$ increases, while $K_{I I}$ decreases. The reason for this is that frictional resistance and the kinematic constraint provided by the jagged surfaces resist crack sliding displacements and therefore reduce $K_{I I}$. The dilatancy, on the other hand, produces a normal gap between the crack surfaces, therefore increasing $K_{I}$.

The curves in figures 4 to 6 have been terminated at the values of $\tau_{\infty}$ that result in crack initiation according to the maximum principal stress cri- 
terion (ref. 15), which postulates that crack growth will occur in a direction perpendicular to the maximum principal stress $\sigma_{p}$, when this value is equal to the value of $\sigma_{p}$ in an equivalent Mode-I case. The angle at which the crack will extend with respect to the horizontal is given by reference 15 .

$$
\left(\tan \frac{\theta}{2}\right)=0.25 \frac{k_{I}}{k_{I I}}-0.25\left(\frac{k_{I}^{2}}{k_{I I}^{2}+8}\right)^{1 / 2}
$$

and the fracture criterion is

$$
K_{I C}=K_{I} \cos ^{3} \frac{\theta}{2}-3 K_{I I} \cos ^{2} \frac{\theta}{2} \sin \frac{\theta}{2}
$$

where $K_{I c}$ is the fracture toughness.

Figures 7 to 9 are plots of the fracture stress for several materials as functions of $\alpha$. The angle of extension $\theta$ is also shown on these figures. As seen from these curves, the effect of tortuosity and friction is to increase the fracture stress and to decrease the angle of extension. In order to separate the effects of friction from those of tortuosity, the case of steel was analyzed for various coefficients of friction. It can be seen from figure 7 that friction has a significant effect on the fracture stress. When $\alpha=40^{\circ}$ and $\mu=0.0$, the increase in fracture stress with respect to zero tortuosity is approximately 20 percent, while for $\mu=0.2$ this increase is 40 percent, and for $\mu=0.5$, it is approximately 90 percent.

\section{DISCUSSION}

From the results obtained in this investigation, it can be concluded that friction and tortuosity have a significant effect on crack tip stress fields when a crack is subjected to mixed mode loading. In the example problems solved in this paper the increase in fracture stress (as predicted from the maximum principal stress theory) with respect to zero friction and tortuosity ranged from 3 to 100 percent. The crack extension angles as predicted from the maximum principal stress theory were also affected by the Mode-I stress intensity which arises from the dilatancy of the interface between opposing crack surfaces.

Although the use of the compliance matrix developed in section 3 renders the numerical problem very attractive, it is limited to simple geometries. The effects of tortuosity and friction in problems with arbitrary geometries can be conveniently analyzed using the constitutive law in conjunction with the finite element method or boundary element method. 


\section{REFERENCES}

1. Clech, J.P.; Keer, L.M.; and Lewis, J.L.: A Model of Tension and Compression Cracks with Cohesive Zone at a Bone-Cement Interface. $\mathrm{J}$. Biomech. Eng., vol. 107, no. 2, May 1985, pp. 175-182.

2. Bazant, Z.P.; and Gambarova, P.: Rough Cracks in Reinforced Concrete. ASCE J. Struct. Div., vol. 106, no. 4, Apr. 1980, pp. 819-842.

3. Jimenez-Perez, R.; Gergely, P.; and White, R.N.: Shear Transfer Across Cracks in Reinforced Concrete. Report 78-4, NSF/RA-780328, Corne 11 University, 1978. (Avail. NTIS as PB-288885/7GA)

4. Fenwick, R.C.; and Paulay, 1.: Mechanisms of Shear Resistance of Concrete Beams. ASCE J. Struct. Div., vol. 94, no. 10, 0ct. 1968, pp. 2325-2350.

5. Ingraffea, A.R.; and Gerstle, W.H.: Non-Linear Fracture Models for Discrete Crack-Propagation. Application of Fracture Mechanics to Cementitious Composites, S.P. Shah Editor, Martinus Nijhoff, 1985, pp. $247-285$.

6. Riggs, H.R.; and Powell, G.H.: Rough Crack Model for Analys is of Concrete. J. Eng. Mech., vol. 112, no. 5, May 1986, pp. 448-464.

7. Plesha, M.E.: Constitutive Models for Rock Discontinuities With Dilatancy and Surface Degradation. Int. J. Numer. Analyt. Methods Geomech., in press, 1981.

8. Bowden, F.P.; and Tabor, D.: The Friction and Lubrication of Solids-Part II, Oxford University Press, 1965.

9. Courtney-Pratt, J.S.; and Eisner, E.: The Effect of a Tangential Force on the Contact of Metaliic Bodies. Proc. R. Soc. London A, vol. 238, no. 1215, Jan. 29, 1951, pp. 529.550.

10. Goodman, R.E.; Heuze, F.E.; and Ohnishi, Y.: Research on Strength-Deformability-Water Pressure Relationships for Faults in Direct Shear. University of California, Berkeley, 1972. (Avail. NTIS, AD-7476/3)

11. Fredriksson, B.: On Elastic Contact Prablems With Friction. Linkoping Studies in Science and Technology, Dissertation No. 6, Linkoping Institute, Sweden, 1976.

12. Bazant, Z.P.; and Gambarova, P.: ASCE J. Struct. Div., vol. 106, no. 4, Apr. 1980, pp. 297-315.

13. Gerasoulis, A.: Use of Piecewise Quadratic Polynominals for the Solution of Singular Integral Equations: of Cauchy Type. Comput. Math. Appl., vol. 8, no. 1, 1982, pp. 15-22.

14. Ballarini, R.: Compliance Matrices for Cracked Bodies. Int. J. Fract., vol. 31, no. 4, Aug. 1986, pp. R63-R66.

15. Broek, D.: Elementary Engineering Fracture Mechanics, 3rd ed., Martinus Nijhoff, 1982. 
TABLE I.

\begin{tabular}{|c|c|c|c|c|}
\hline & $\underset{\text { ksi-in1/2 }}{K_{I c}}$ & $\begin{array}{r}E, \\
k s i\end{array}$ & $v$ & $\boldsymbol{\mu}$ \\
\hline $\begin{array}{l}\text { M50 Steel } \\
\text { Concrete } \\
\text { Sintered Stlicon } \\
\text { Carbide Ceramic }\end{array}$ & $\begin{array}{r}18 \\
1 \\
4\end{array}$ & $\begin{array}{rr}30 & 000 \\
3 & 000 \\
59 & 000\end{array}$ & $\begin{array}{l}0.3 \\
.2 \\
.14\end{array}$ & $\begin{array}{r}0.20 \\
.50 \\
.30\end{array}$ \\
\hline
\end{tabular}




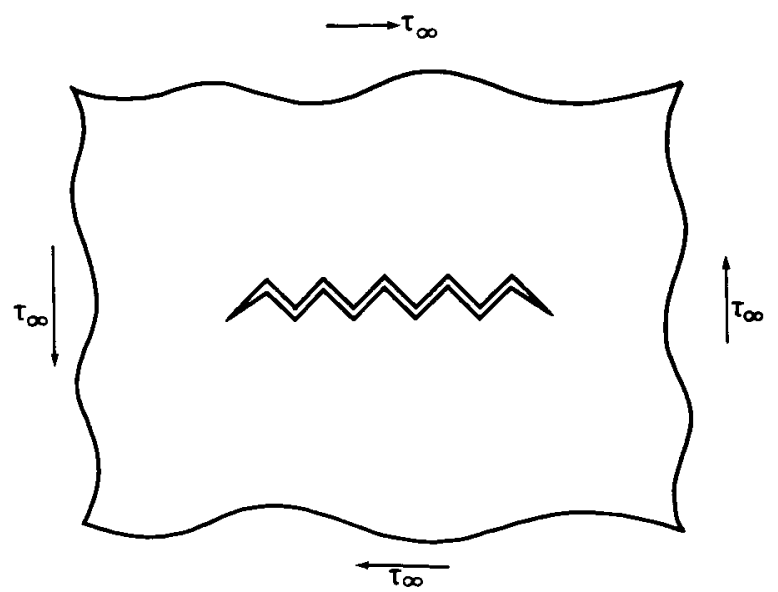

(A) GLOBALLY SMOOTH, BUT MICROSCOPICALLY ROUGH CRACK IN AN INFINITE MEDIUM.

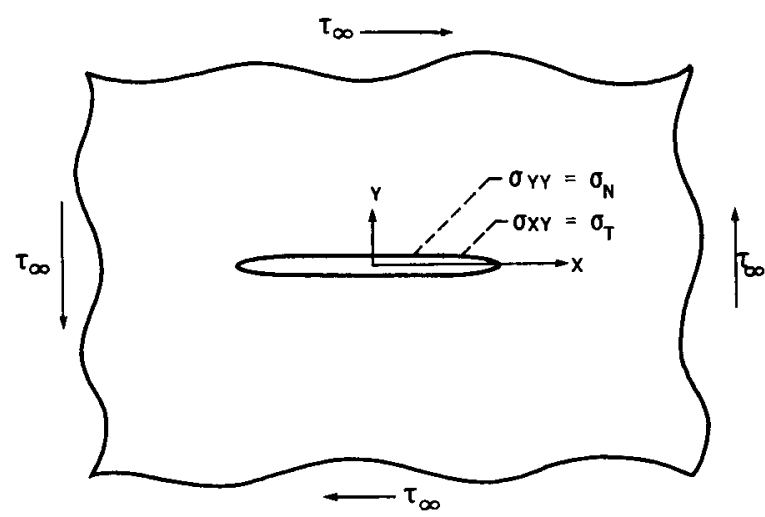

(B) MATHEMATICAL IDEALIZATION.

FIGURE 1. - PROBLEM CONFIGURATION.

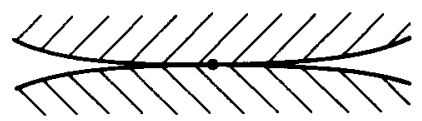

(A) GLOBALLY SMOOTH CONTACT SURFACE.

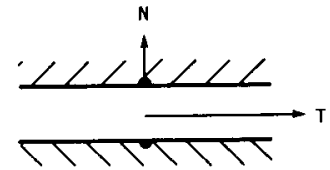

(B) LOCAL TANGENT-NORMAL COORDINATE SYSTEM AT A CONTACT POINT PAIR (SURFACES ARE SHOWN SEPARATED FOR CLARITY AND ROUGHNESS IS NOT SHOWN).

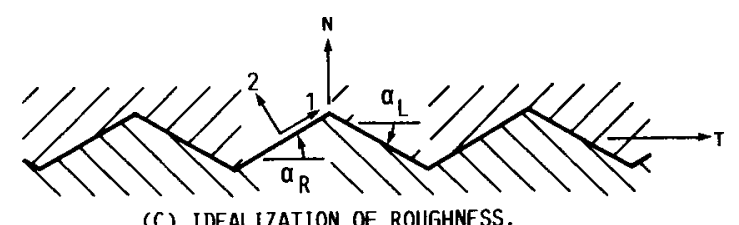

(C) IDEALIZATION OF ROUGHNESS.

FIGURE 2. - TWO-DIMENSIONAL CONTACT PROBLEM. 

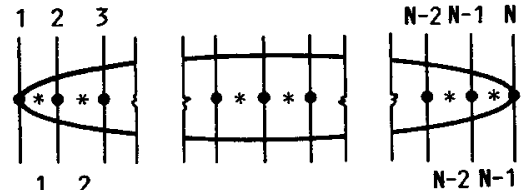

(A) LOCATIONS AS OBTAINED FROM [13].
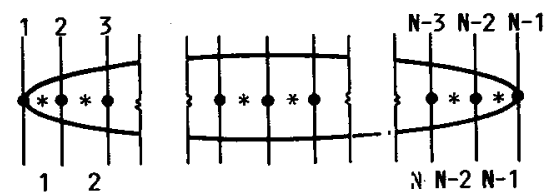

(B) MODIFIED LOCATIONS PROPOSED IN SECTION 4.

FIGURE 3. - LOCATION OF INTEGRATION AND COLLOCATION POINTS.

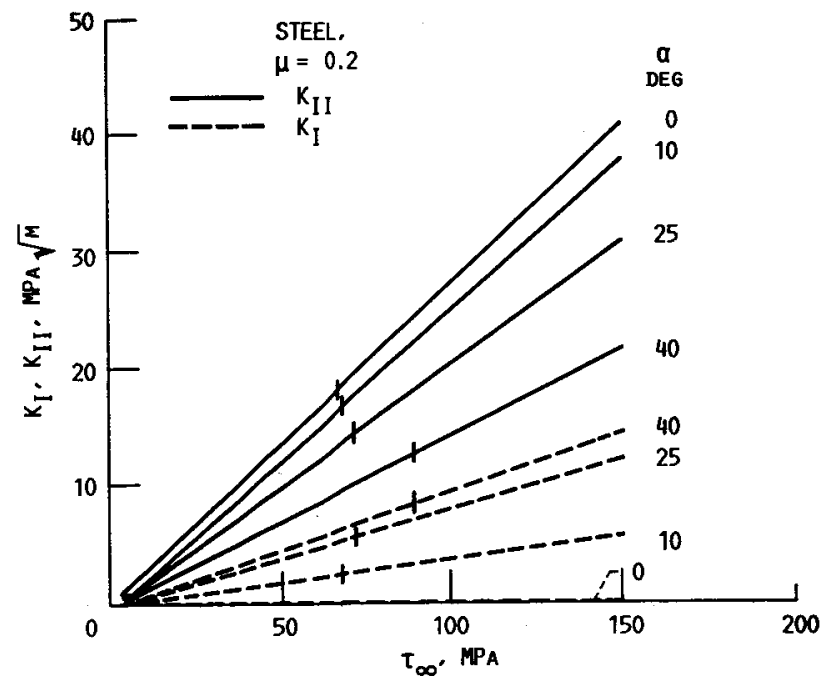

FIGURE 4. - STRESS INTENSITY FACTORS AS FUNCTION OF APPLIED LOADING (STEEL). 


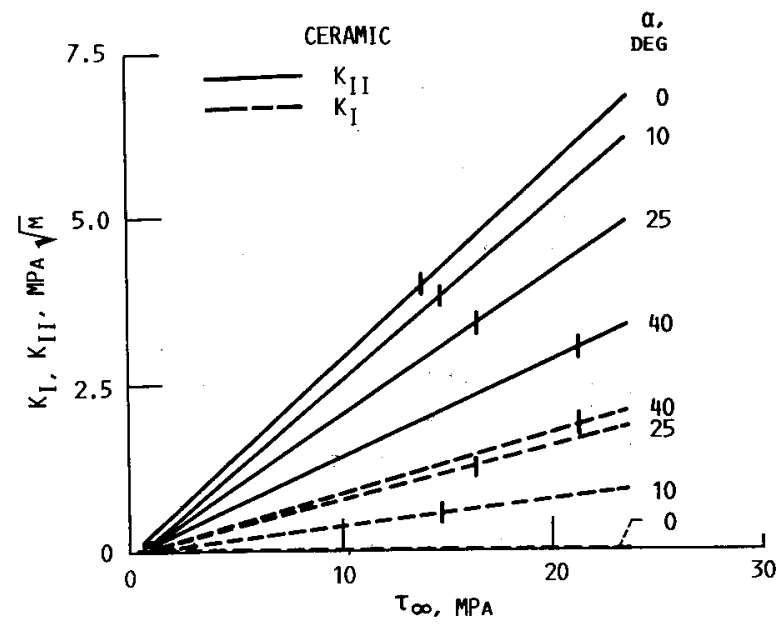

FIGURE 5. - STRESS INTENSITY FACTORS AS FUNCTIONS OF APPLIED LOADING (CERAMIC).

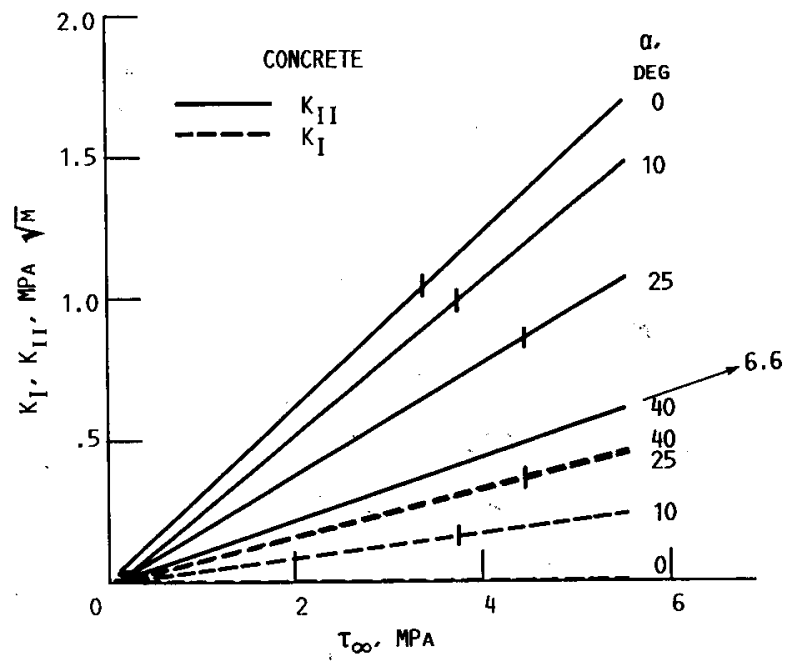

FIGURE 6. - STRESS INTENSITY FACTORS AS FUNCTIONS OF APPLIED LOADING (CONCRETE). 


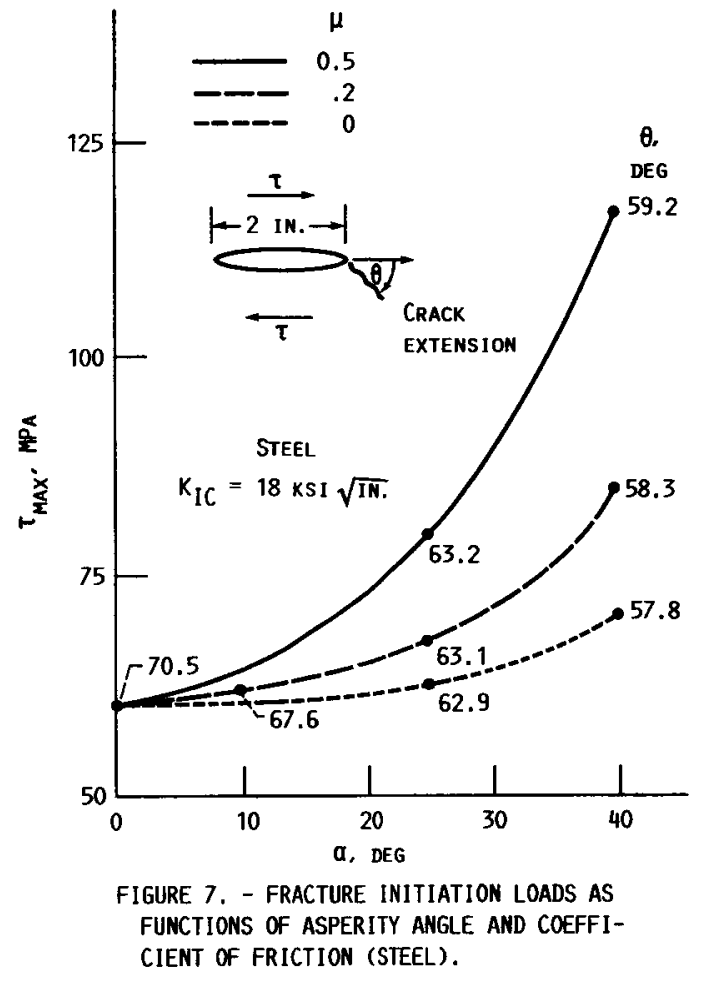




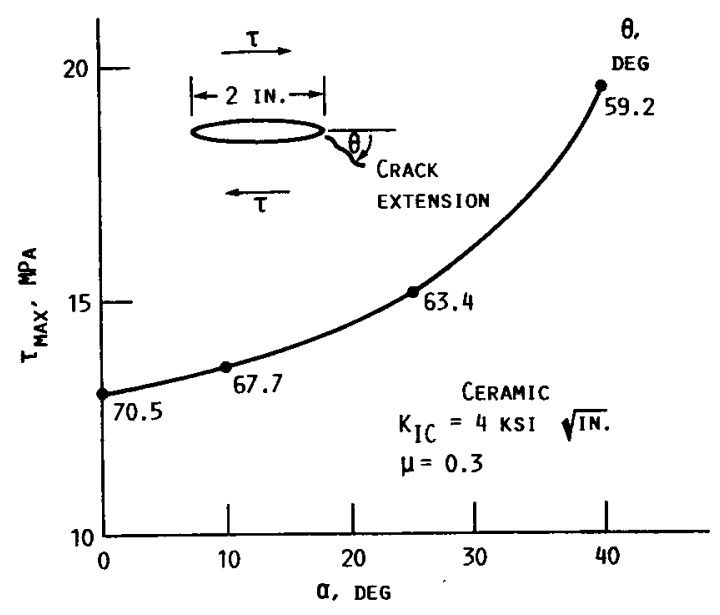

FIGURE 8. - FRACTURE INITIATION LOAD AS

FUNCTION OF ASPERITY ANGLE (CERAMIC).

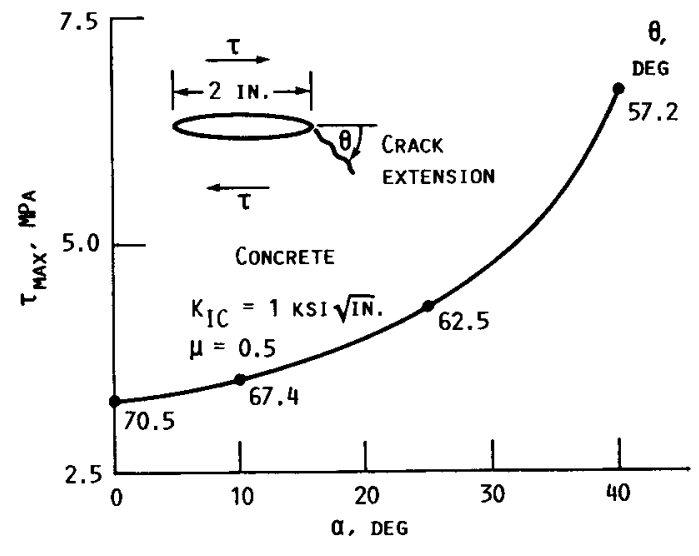

FIGURE 9. - FRACTURE INITIATION LOAD AS

FUACTION OF ASPERITY ANGLE (CONCRETE). 


\begin{tabular}{|c|c|c|}
\hline 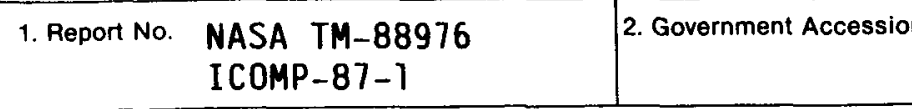 & 2. Government Accession No. & 3. Recipient's Catalog No. \\
\hline \multirow{2}{*}{\multicolumn{2}{|c|}{$\begin{array}{l}\text { 4. Title and Subtitle } \\
\text { The Effects of Crack Surface Friction and Roughness } \\
\text { on Crack Tip Stress Fields }\end{array}$}} & $\begin{array}{l}\text { 5. Report Date } \\
\text { February } 1987\end{array}$ \\
\hline & & $\begin{array}{l}\text { 6. Performing Organization Code } \\
553-13-00\end{array}$ \\
\hline \multirow{2}{*}{\multicolumn{2}{|c|}{$\begin{array}{l}\text { 7. Author(s) } \\
\text { Roberto Ballarini and Michael E. Plesha }\end{array}$}} & $\begin{array}{l}\text { 8. Performing Organization Report No. } \\
\text { E-3445 }\end{array}$ \\
\hline & & 10. Work Unit No. \\
\hline \multirow{2}{*}{\multicolumn{2}{|c|}{$\begin{array}{l}\text { 9. Performing Organization Name and Address } \\
\text { National Aeronautics and Space Administration } \\
\text { Lewis Research Center } \\
\text { Cleveland, Ohio } 44135\end{array}$}} & \\
\hline & & \begin{tabular}{|l} 
\\
13. Type of Report and Period Covered
\end{tabular} \\
\hline \multicolumn{2}{|l|}{ 12. Sponsoring Agency Name and Address } & Technical Memorandum \\
\hline \multicolumn{2}{|l|}{$\begin{array}{l}\text { National Aeronautics and Space Administration } \\
\text { Washington, D.C. } 20546\end{array}$} & 14. Sponsoring Agency Code \\
\hline \multicolumn{3}{|c|}{$\begin{array}{l}\text { 15. Supplementary Notes } \\
\text { Roberto Ballarini, Case Western Reserve University, Department of Civil Engineering, Cleveland, } \\
\text { Ohio } 44106 \text { (work funded by Cooperative Agreement NCC 3-46). Michael E. Plesha, Institute for } \\
\text { Computational Mechanics in Propulsion, NASA Lewis Research Center (work funded under Space Act } \\
\text { Agreement C99066-G and U.S. Army Research Office Grant DAAL03-86-K-0134); presently at University of } \\
\text { Wisconsin, Department of Engineering Mechanics, Madison, Wisconsin 53706. }\end{array}$} \\
\hline \multicolumn{3}{|c|}{$\begin{array}{l}\text { 16. Abstract } \\
\text { A model is presented which can be used to incorporate the effects of friction and } \\
\text { tortuosity along crack surfaces through a constitutive law applied to the inter- } \\
\text { face between opposing crack surfaces. The problem of a crack with a saw-tooth } \\
\text { surface in an infinite medium subjected to a far-field shear stress is solved and } \\
\text { the ratios of Mode-I stress intensity to Mode-II stress intensity are calculated } \\
\text { for various coefficients of friction and material properties. The results show } \\
\text { that tortuosity and friction lead to an increase in fracture loads and alter the } \\
\text { direction of crack propagation. }\end{array}$} \\
\hline 17. Key Words (Suggested by Author(S)) & \multirow{2}{*}{\multicolumn{2}{|c|}{$\begin{array}{l}\text { 18. Distribution Statement } \\
\text { Unclassified - unlimited } \\
\text { STAR Category } 39\end{array}$}} \\
\hline $\begin{array}{l}\text { Crack surface friction; Crack surface } \\
\text { roughness; Mixed mode fracture; Interface } \\
\text { constitutive law }\end{array}$ & & \\
\hline $\begin{array}{l}\text { 19. Security Classif. (of this report) } \\
\text { Unc lass if ied }\end{array}$ & sified & \begin{tabular}{|c|c|} 
21. No. of Dages & 22. Price* \\
17 & $\mathrm{AO2}$
\end{tabular} \\
\hline
\end{tabular}

\footnotetext{
"For sale by the National Technical Information Service, Springfield, Virginia 22161
} 